\title{
Contamination Métallique D'une Espèce De Poisson (Brama Brama) De La Côte Dakaroise
}

\author{
Cheikh Tidiane Dione, \\ Ibrahima Diagne, \\ Momar Ndiaye,
}

Faculte des Sciences et Techniques, Laboratoire de Chimie Physique

Organique et d'Analyse Environnementale (LCPOAE)-UCAD,

Dakar, Sénégal

Cheikhna Diebakate,

Faculte des Sciences et Techniques,

Département de Biologie Animale, UCAD, Dakar, Sénégal

\section{Birame Ndiaye,}

Abdoulaye Diop,

Faculte des Sciences et Techniques, Laboratoire de Chimie Physique

Organique et d'Analyse Environnementale (LCPOAE)-UCAD,

Dakar, Sénégal

Doi: 10.19044/esj.2018.v14n12p374 URL:http://dx.doi.org/10.19044/esj.2018.v14n12p374

\begin{abstract}
Pollution of metal origin constitutes one of the major risks in the world today. The metal elements can be very dangerous to human health when they are present in the environment at high concentrations. Thus, the evaluation of the metal contamination in marine organisms, especially fish, makes it possible to predict a possible contamination of humans. This paper focuses on studying the metal contamination of the beach of Soumbédioune, located at the western frontage of the area of Dakar. This site, where fish products are offloaded, is the home to Canal IV (West Canal), which drains urban wastewater. In this study, we evaluated the concentrations of the metal elements $\left(\mathrm{Fe}, \mathrm{Zn}^{2+}, \mathrm{Cr}^{6+}\right)$ in the bodies of Brama brama using a visible spectrophotometry UV. The results obtained show a strong concentration of chromium plates of $24,5 \mu \mathrm{g} / \mathrm{g}$ which is obtained at the level of the liver. Zinc presents a content of $16,7 \mu \mathrm{g} / \mathrm{g}$ at the level of the skin, but it was not detected at the level of the liver and the flesh. The maximum iron content was recorded at the level of the liver with a value of $77,5 \mu \mathrm{g} / \mathrm{g}$. However, the values obtained were definitely higher than the standards established by FAO and the CEE.
\end{abstract}


Keywords: Contamination, Brama brama, Soumbédioune, spectrophotometry, iron, chromium, zinc

\section{Résumé}

Les pollutions d'origine métallique constituent l'un des risques majeurs dans le monde actuel. Les éléments métalliques peuvent être très dangereux pour la santé humaine lorsqu'ils sont présents dans l'environnement à des concentrations élevées. Ainsi l'évaluation de la contamination métallique dans les organismes marins, notamment les poissons, permet de prévoir une possible contamination de l'homme. Ce travail consiste à étudier la contamination métallique de la plage de Soumbédioune, située sur la façade ouest de la région de Dakar. Ce site, lieu de débarquement de produits halieutiques, abrite le canal IV (canal ouest) qui draine des eaux de rejets urbaines. Nous avons évalué les concentrations des éléments métalliques ( $\mathrm{Fe}, \mathrm{Zn}^{2+}, \mathrm{Cr}^{6+}$ ), dans les organes du Brama brama par la spectrophotométrie UV- visible. Les résultats obtenus montrent une forte concentration en chrome qui est de $24,5 \mu \mathrm{g} / \mathrm{g}$ obtenue au niveau du foie. Le zinc présente une teneur de $16,7 \mu \mathrm{g} / \mathrm{g}$ au niveau de la peau mais il n'est pas détecté au niveau du foie et de la chair. La teneur maximale en fer est enregistrée au niveau du foie avec une valeur de 77,5 $\mu \mathrm{g} / \mathrm{g}$. En effet, les valeurs trouvées sont nettement supérieures aux normes établies par la FAO et la CEE.

Mots clés : Contamination, Brama brama, Soumbédioune,
spectrophotométrie, fer, chrome, zinc

\section{Introduction}

Les problèmes posés par la dispersion des polluants dans l'environnement suscitent l'intérêt de la communauté scientifique depuis un certain nombre d'années. En effet, la protection de l'environnement a lieu grâce à la connaissance du destin de ces polluants dans l'environnement et de leurs effets sur les écosystèmes aquatiques (Atolage \& Arenu, 2007).

Parmi les contaminants majeurs de l'environnement, les métaux lourds posent de sérieux problèmes écologiques par leurs toxicités et leur bioaccumulation potentielle dans plusieurs espèces aquatiques, induisant des effets dévastateurs sur la balance écologique de l'environnement aquatique.

Les métaux lourds sont des polluants engendrés par l'activité humaine ayant un fort impact toxicologique. Il existe de nombreux métaux toxiques comme le cadmium, le plomb et le mercure. Ils ont des impacts sur les végétaux, les produits de consommation courante et sur l'homme. Les poissons concentrent les éléments indispensables pour leur vie, mais aussi les contaminants dont les métaux lourds qui sont 1'objet de notre étude. Certains de ces métaux existent à l'état naturel. Mais d'autres sont déversés directement 
dans le milieu aquatique des côtes dakaroises par la combustion du carburant, les déchets industriels et les eaux usées (Canli \& Kalay, 1998).

Il s'est avéré que les poissons sont des vecteurs de contamination aux métaux lourds chez l'homme, de sorte que certaines espèces servent aujourd'hui d'outils biologiques à l'évaluation de la pollution des eaux par les métaux lourds. Il n'est pas donc surprenant que de nombreuses études aient été menées sur ce type de pollution chez les différentes espèces de poissons. Effectivement, les organismes marins en particulier les poissons sont capables grâce à leur métabolisme d'accumuler des éléments contaminants de l'environnement dans certains de leurs organes.

Il est de la plus haute importance de noter que, bien que les muscles fournissent relativement une faible teneur en métaux du fait de leur faible activité métabolique, ils peuvent servir d'indicateur biologique circonstanciel en raison de sa masse par rapport aux autres organes comme le foie et les branchies (Chaffai Nee Hamza, 1993).

A partir d'une certaine concentration spécifique de chaque métal lourd dans le muscle, l'homme est exposé à la contamination par les poissons.

Le chrome ( $\mathrm{Cr}$ ) est un élément chimique de masse atomique relativement élevée qui est toxique même à faible dose, surtout s'il a un effet cumulatif par ingestion répétée de la nourriture.

Le cuivre $(\mathrm{Cu})$ est essentiel pour le métabolisme des poissons, mais dangereux pour l'homme. Il a été montré que même dans les eaux non polluées par le cuivre les poissons l'accumulent.

Le fer $(\mathrm{Fe})$ est essentiel pour la respiration cellulaire chez les animaux. C'est un puissant catalyseur de certaines réactions biochimiques, cependant il est toxique à des doses élevées.

L'effet toxique du plomb $(\mathrm{Pb})$ dépend du cycle de vie du poisson, du $\mathrm{pH}$, de la dureté de l'eau et de la présence des matières organiques. Le $\mathrm{Pb}$ est classé parmi les métaux les plus toxiques pour l'homme et pour les animaux. Il ne montre pas d'accumulation le long de la chaine alimentaire pour les organismes marins. Il n'a aucun rôle connu dans les systèmes biologiques.

Le zinc ( $\mathrm{Zn}$ ) est un nutriment essentiel pour les organismes aquatiques, mais s'il est présent en excès, il peut devenir un contaminant environnemental (Karine Noppe, 1995).

Ces éléments sont des polluants particulièrement dangereux pour la santé humaine. Leur toxicité est renforcée par le phénomène de bioaccumulation et de bioconcentration dans l'organisme. Certainement, , ces éléments pénètrent dans l'organisme soit par ingestion à travers la chaine alimentaire, soit par inhalation (Diagne, 2012).

Le but de cette étude est de déterminer les concentrations des métaux $\left(\mathrm{Fe}, \mathrm{Zn}^{2+}, \mathrm{Cr}^{6+}\right)$ dans la chair, la peau et le foie d'une espèce de poisson (Brama brama) en vue d'évaluer le niveau de contamination de nos côtes. 


\section{Matériel et méthode}

\section{Site d'étude}

La région de Dakar constitue un pôle économique important au Sénégal par sa forte concentration d'industries légères et lourdes situées au bord de la mer. Cette région comporte plusieurs dépressions humides dont les côtes. C'est dans cette optique que nous nous sommes intéressés à l'évaluation de la bioaccumulation métallique des poissons de la baie de Soumbédioune.

La plage de Soumbédioune est située au quartier de la Médina de Dakar sur la corniche ouest (Figure 1). C'est un lieu de débarquement des produits halieutiques qui reçoit les eaux usées provenant du canal ouest qui traverse une partie de la région de Dakar.

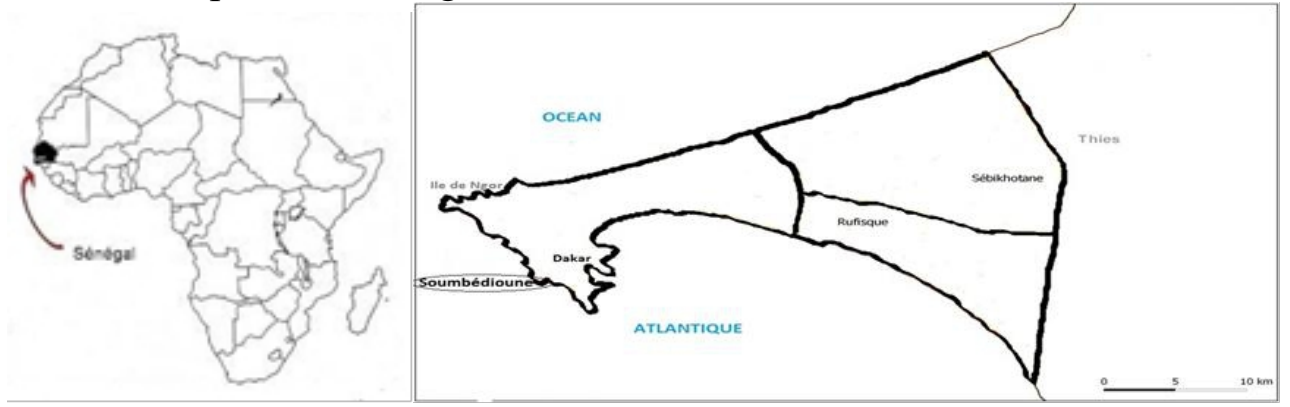

Figure 1. Site de prélèvements Echantillonnage

L'échantillonnage constitue une étape cruciale de notre étude. Le but visé est d'obtenir un éventail d'échantillons représentatifs qui reflète toutes les caractéristiques physiques et chimiques du site étudié. Les principales étapes qui caractérisent une démarche d'échantillonnage sont l'élaboration d'une stratégie de prélèvements d'échantillons sur les sites et leurs conservations.

Les échantillons de poissons ont été récoltés au niveau de la baie de Soumbédioune. Ils sont transportés au laboratoire puis conservés au réfrigérateur à $-20^{\circ} \mathrm{C}$ avant la préparation.

\section{Préparation des échantillons}

$\mathrm{Au}$ laboratoire, nous avons disséqué les poissons; puis la peau, la chair et le foie ont été prélevés et séchés à l'étuve pendant deux heures à $60^{\circ} \mathrm{C}$ jusqu'à ce que le poids ne varie plus et enfin les échantillons ont été broyés et conservés dans des sachets en plastiques.

\section{Extraction des métaux}

Pour la minéralisation, trois prises de $0,5 \mathrm{~g}$ de la poudre obtenue sont attaquées avec $4 \mathrm{ml}$ d'acide nitrique $\mathrm{HNO}_{3}(14 \mathrm{~N})$ dans des capsules en verre puis placées à l'étuve à $80^{\circ} \mathrm{C}$ pendant une heure pour la digestion du tissu et l'évaporation totale de l'acide. Le produit de la digestion est récupéré avec quelques $\mathrm{ml} \mathrm{d}$ 'eau distillée et repris dans une fiole jaugée de $50 \mathrm{ml}$ en ajoutant 
de l'eau distillée. La solution ainsi obtenue est utilisée pour la lecture au photomètre PF-11.

\section{Méthode d'analyse}

L'analyse des éléments métalliques $\left(\mathrm{Fe}, \mathrm{Zn}^{2+}\right.$ et $\left.\mathrm{Cr}^{6+}\right)$ dans le foie, la chair et la peau du Brama brama a été réalisée au Laboratoire de Chimie Physique Organique et d'Analyse Environnementale (LCPOAE) de la Faculté des Sciences et Techniques de l'Université Cheikh Anta Diop de Dakar.

Les analyses ont été réalisées par la spectrophotométrie UV-visible à l'aide du photomètre PF-11 (Auger, 1989).

\section{Résultats et discussion}

Les résultats des analyses des teneurs moyennes des métaux lourds, pour une série de trois mesures dans les organes du Brama brama et leurs écarts type, sont regroupés dans le Tableau 1.

Tableau 1. Les teneurs des métaux étudiés en $\mu \mathrm{g} / \mathrm{g}$ de poids sec dans les organes du Brama brama

\begin{tabular}{|c|c|c|c|}
\hline Organes & Foie & Peau & Chair \\
\hline Elément & $77,5 \pm 2,1$ & $21 \pm 1,4$ & $72 \pm 2,8$ \\
\hline$[\mathrm{Fe}] \pm \sigma$ & $24,5 \pm 0,7$ & $14,5 \pm 0,7$ & $9,3 \pm 3,5$ \\
\hline$\left[\mathrm{Cr}^{6+}\right] \pm \sigma$ & $\mathrm{ND}$ & $16,7 \pm 1,5$ & $\mathrm{ND}$ \\
\hline$\left[\mathrm{Zn}^{2+}\right] \pm \sigma$ & &
\end{tabular}

Le chrome enregistre des concentrations qui varient entre 9,3 $\mu \mathrm{g} / \mathrm{g}$ au niveau de la chair et $24,5 \mu \mathrm{g} / \mathrm{g}$ au niveau du foie.

Pour le fer, les teneurs sont plus élevées et varient entre $21 \mu \mathrm{g} / \mathrm{g}$ au niveau de la peau et $77,5 \mu \mathrm{g} / \mathrm{g}$ au niveau du foie.

Le zinc enregistre une teneur de $16,7 \mu \mathrm{g} / \mathrm{g}$ au niveau de la peau mais il n'est pas détecté dans le foie et dans la chair.

\section{Accumulation des métaux dans les différents organes}

Les Figures 2, 3 et 4 présentent les teneurs (en $\mu \mathrm{g} / \mathrm{g})$ du chrome, du fer et du zinc, respectivement, dans les différents organes. 


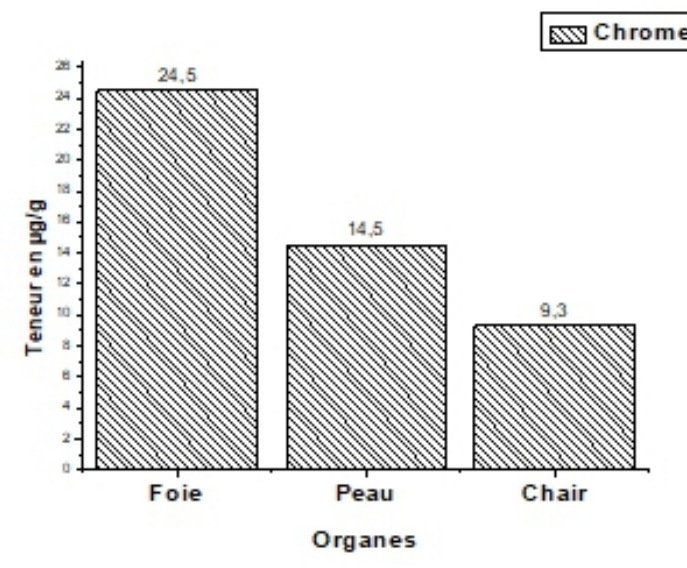

Figure 2. Teneurs en chrome en $\mu \mathrm{g} / \mathrm{g}$ dans les organes du Brama

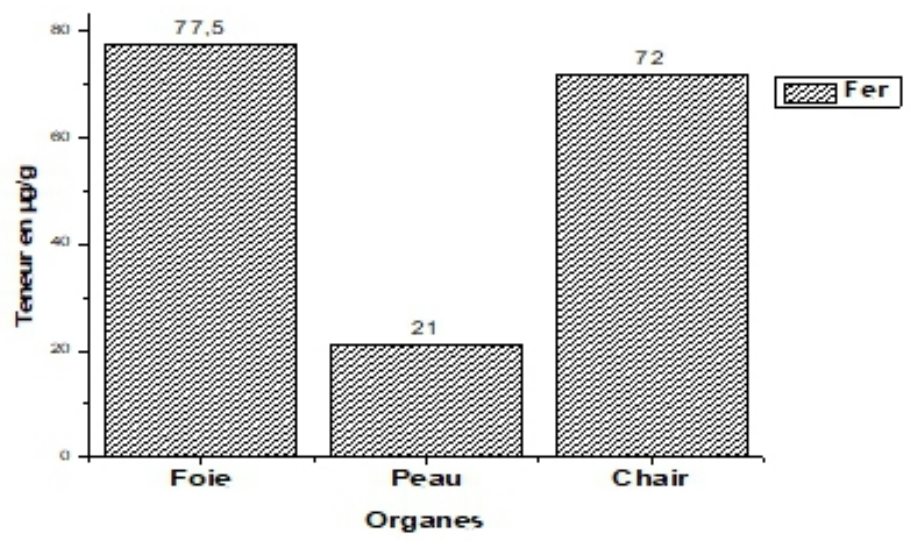

Figure 3. Teneurs en fer en $\mu \mathrm{g} / \mathrm{g}$ dans les organes du Brama.

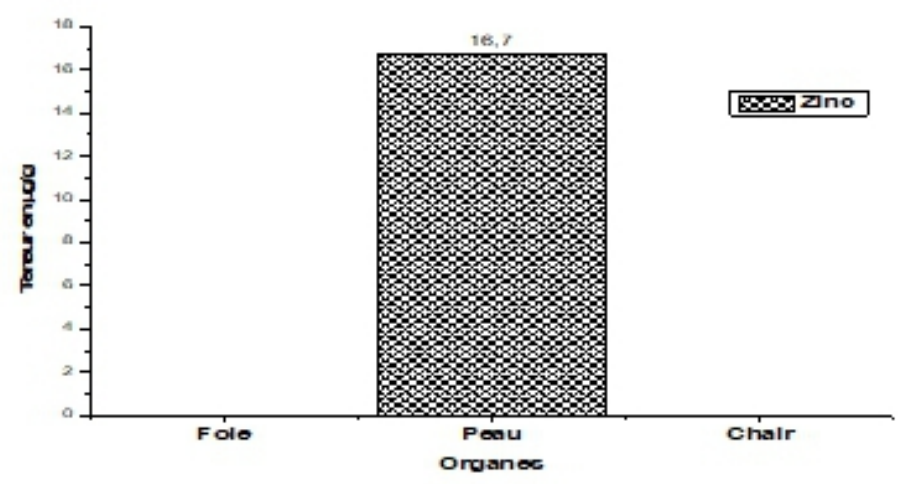

Figure 4. Teneurs en zinc en $\mu \mathrm{g} / \mathrm{g}$ dans les organes du Brama. 
L'analyse de la Figure 2, montre que les teneurs en chrome varient d'un organe à l'autre.

Le foie enregistre la teneur la plus élevée avec $24,5 \mu \mathrm{g} / \mathrm{g}$ suivi de la peau avec $14,5 \mu \mathrm{g} / \mathrm{g}$ et de la chair avec $9,3 \mu \mathrm{g} / \mathrm{g}$ de poids sec. Ces valeurs obtenues dépassent la valeur limite qui est de $5,5 \mu \mathrm{g} / \mathrm{g}$ de poids sec.

La Figure 3 dévoile que le foie abrite aussi la teneur en fer la plus élevée avec $77,5 \mu \mathrm{g} / \mathrm{g}$ suivi de la chair avec $72 \mu \mathrm{g} / \mathrm{g}$ et de la peau avec $21 \mu \mathrm{g} / \mathrm{g}$ de poids sec. Ces valeurs obtenues dépassent la norme qui est de $20 \mu \mathrm{g} / \mathrm{g}$ de poids sec.

L'analyse de la Figure 4, montre que la peau enregistre la valeur la plus élevée avec $16,5 \mu \mathrm{g} / \mathrm{g}$ de poids sec. Cependant il n'est pas détecté au niveau de la chair et du foie.

Le fer reste le principal contaminant métallique et montre une large distribution dans le foie. Ce métal a tendance à se concentrer facilement dans le foie. La faible teneur obtenue dans le muscle est significative car le muscle est un organe de métabolisation (Mohamed et al., 2013).

Nous avons observé des teneurs plus élevées des métaux dans la peau et le foie que dans la chair. Les différences des concentrations entre les organes varient selon un ordre de grandeur. Ces variations peuvent provenir des fonctions physiologiques des différents organes. Ainsi, la peau, organe externe en contact direct avec l'eau, joue un rôle primordial dans les phénomènes respiratoires des poissons. En cas de pollution, les processus respiratoires risquent d'être perturbés. Le foie est souvent recommandé comme un organe cible lors du contrôle des concentrations en métaux dans les milieux aquatiques. Cependant, le muscle est généralement analysé car c'est la partie principale du poisson consommée par les humains et elle est impliquée dans les risques sanitaires (El Morhit et al., 2013).

Le foie semble être plus sensible aux conditions environnementales, même si la sensibilité aux métaux semble être variable pour les différents éléments métalliques dans les différents organes de poissons étudiés.

Le $\mathrm{Zn}$ joue aussi un rôle important dans la santé humaine et la santé des écosystèmes car il constitue un élément essentiel (à l'état de traces $<0.01 \%$ ) pour le bon fonctionnement du métabolisme des organismes vivants (Tahiri, 2005). En effet, il agit à titre de cofacteur dans les métallo-enzymes en catalysant la réaction enzymatique ou en assurant une structure tridimensionnelle active de l'enzyme. Il est aussi un élément essentiel au bon fonctionnement de tout organisme vivant. Il intervient dans l'activité de nombreuses enzymes et il est indispensable à un grand nombre de fonctions ou de situations physiologiques, aussi diverses que la croissance et la multiplication cellulaire, le métabolisme osseux, la cicatrisation des blessures, la reproduction et la fertilité, l'immunité et la gustation, le fonctionnement cérébral et la protection contre les radicaux libres (El Morhit et al., 2012). 
Le fer est un oligo-élément indispensable pour la croissance. Il facilite le transport de l'oxygène.

Le fer $(\mathrm{Fe})$ est essentiel pour la respiration cellulaire chez les animaux. C'est un puissant catalyseur de certaines réactions biochimiques ; cependant il est toxique à des doses élevées.

La contamination métallique des poissons de la baie de Soumbédioune peut être due aux déversements des eaux usées du canal ouest, des rejets d'eaux fécales urbaines et des déchets solides ménagers rejetés directement en mer par les riverains.

\section{Ordre d'accumulation des métaux dans les différents organes}

L'accumulation des métaux lourds $\left(\mathrm{Fe}, \mathrm{Zn}\right.$ et $\left.\mathrm{Cr}^{6+}\right)$ dans le brama brama varie d'un organe à un autre. Par conséquent, on peut établir un ordre d'accumulation (O.A) des éléments métalliques dans les différents organes.

Pour le fer, on a l'ordre d'accumulation dans les différents organes étudiés (O.A) : $\quad$ foie $>$ chair $>$ peau.

Quant au chrome, on constate l'ordre d'accumulation suivant (O.A) : foie $>$ peau $>$ chair.

Le zinc n'est accumulé que dans la peau, O.A : peau $>$ au foie et la chair.

D'après cet ordre d'accumulation, on peut dire que la teneur en fer dans le foie est supérieure à celle de la chair et trois fois supérieure à celle de la peau. Quant au chrome, la teneur dans le foie est supérieure à celle de la chair et deux fois supérieure à celle de la peau. Pour le zinc, la teneur de la peau est très supérieure à celle de la chair et du foie.

\section{Conclusion}

La pollution de l'environnement par les métaux lourds (fer, chrome, zinc, cuivre, nickel, mercure, arsenic et cadmium) est due à des émissions provenant des activités humaines.

Dans le cadre de ce travail, nous avons établi le profil de contamination des côtes de la région de Dakar, par le dosage des métaux lourds dans les organes du Brama brama. Les analyses ont été effectuées par la spectrophotométrie UV-visible à l'aide du photomètre PF-11.

Les résultats obtenus, à partir du bio-indicateur biologique, permettent une évaluation de l'état de contamination par le fer, le zinc et le chrome du littoral dakarois. Une quantification de l'impact des apports anthropiques de ces métaux dans le milieu aquatique a été également réalisée.

Les fortes teneurs des métaux trouvées dans les organes du Brama brama, ont révélé le danger réel que présente cette partie du littoral dakarois, laquelle est utilisée pour la pêche et pour le débarquement des produits halieutiques. De plus, la consommation de poissons de cette zone peut causer 
un problème de santé publique.

Il est urgent d'effectuer un contrôle régulier basé sur une politique efficace dont les acteurs seront l'Etat, les industriels et la communauté scientifique.

\section{Remerciements}

Nous tenons à remercier et à exprimer notre profonde gratitude à $\mathrm{M}$. Paul NKENG qui nous a soutenus en apportant du matériel à travers le SAMU environnement de Strasbourg pour l'élaboration de ce document.

\section{References:}

1. Atolage, B.O. \& Arenu, M.O. (2007). Bioaccumulation of some trace elements in the body parts of fish species associated with soil sediment and water from Eoemagania confluence in Nasarawa state, Nigeria. EJEAFChe ; 6, 5, 2001-2008.

2. Auger, D. (1989). Méthode de dosage du cadmium, du cuivre, du plomb et du zinc dans les milieux biologiques. Rapp. Direction Environnement Recherches océaniques(DERO) 07-MB.

3. Canli, M. \& Kalay, O.A.M. (1998). Level of heavy metals (Cd, $\mathrm{Pb}, \mathrm{Cu}$, $\mathrm{Cr}$ and $\mathrm{Ni}$ ) in tissue of Cyprinus carpio, Barbus capito and Chondrostoma regium from the Seyhan River. Turkish journal of zoology, 22, 149-157.

4. Chaffai Nee Hamza (1993). Etude de la bioaccumulation métallique et des méthotionneines chez les poissons de la côte de Sfax pour le sud. Tunisie, 157p. (Maroc) D.S.T, 61,8-17.

5. DEA (1995). hydrologie, hydrogéologie, géostatistiques et géochimie 1995-1996, laboratoire de géologie appliquée .Université PIERRE et MARIE CURIE, PARIS VI .P 92.

6. Diagne, I. (2012). Contamination des moules et des sédiments des cotes de la région de Dakar par certains métaux (cadmium, chrome, plomb) : application au dosage du cadmium, chrome, plomb par la spectrométrie d'émission atomique par arc électrique. DEA, Université CHEIKH ANTA DIOP de Dakar, p58.

7. El Morhit, A. \& Belghity, D. (2013). Contamination métallique de PAGELLUS ACARNE, SARDINA PILCHARDUS ET DIPLODUS VULGARIS de la côte atlantique sud (MAROC), Larhyss Journal, ISSN 1112-3680, $\mathrm{n}^{\circ} 14$, Juin 2013, pp .131-148.

8. El Morhit, M., Fekhaoui, M., El Abidi, A., Yahyaoui A., \& Hamdani, A. (2012). Impact des activités humaines sur la dégradation de la qualité des sédiments de l'estuaire de Loukkos

9. Karine Noppe (1995). Contamination métallique des sédiments des cours d'eau du bassin Artois-Picardie et son impact sur la 
contamination des chairs et des foies de poissons, mémoire de

10. Mohamed, EL., Morhit, M., Fekhaoul, A., El Abidi, A., Yahyaoui, A. (2013). Contamination métallique d'Anguilla anguillal au niveau de l'estuaire de LOUKKOS(Maroc), bulletin de l'institut scientifique, Rabat, section sciences de la vie, 2013, n³5, 111-118.

11. Tahiri, L. (2005). Contamination métallique de Mytilus galloprovincialis et des sédiments au niveau de l'estuaire de Bouregreg (Maroc).Walter qual. Res. J.40 (1).111-119.

12. Thomas Osset. Thèse, contamination chimique des poissons d'eau douce. Bilan sanitaire en France et indicateurs de pollution, Université Claude BERNARD Lyon p76. 